\title{
Towards transparency trends in academic databases?
}

In a recent opinion paper published in the Journal of Informetrics, Franceschini, Maisano, and Mastrogiacomo (2016a) classified database errors found in various studies published in recent years. These studies identified systematic errors in the Web of Science Core Collection and Scopus databases design, primarily the latter. This structured collection of errors is important for developing information retrieval methodologies and indexing processes in bibliographic scientific databases. Here, we would like to comment on certain relevant aspects of the relationship between academic databases and research evaluation.The errors classified by Franceschini et al. (2016a) indicate that in what are considered the two main multidisciplinary databases for evaluating scientific activity, (i) databases are making mistakes in the indexing process of records and (ii)errors significantly affect indicators of scientific activity. Additionally, because of the continuous updating of data already indexed and of the periodic addition of new bibliographic records, it remains unknown whether the identified errors may have affected research evaluations such as bibliometric analyses of scientific disciplines, institutional benchmarks, academic evaluations of researchers, or scientific policies. On the other hand, in the current context,transparency and open data not only are principles used in research evaluations (Hicks, Wouters, Waltman, de Rijcke, \& Rafols, 2015) but also are being valued by several scientific journals and publishers for (a) identifying the review, acceptance and publication date of a scientific paper, (b) specifying the contribution of each author of the manuscript, (c) requiring that all raw data generated in the published study are included as Supplementary material or linked to a repository (Candela, Castelli, Manghi, \& Tani, 2015), and (d) either providing the manuscript reviews alongside the published article, as in F1000 Research and eLife, or showing in each academic editor profile the papers reviewed, as in PeerJ. For these reasons, we hope that both databases, which are crucial to conducting evaluations, can improve transparency with respect to indexing process similarly to the ways of PubMed has been developing in recent years. For example, since1996, PubMed has published the number of errors (like errata, retractions or partial retractions) detected and corrected inthe scientific literature indexed. Also, all records linked by any error or by any other motive (republished, updated versions or duplicates, among others) are linked and classified according to their issue. In the case of duplicates, PubMed uses the MeSH term "Duplicate Publication" [PT] to identify bibliographic records that contain identical or nearly identical content and were published simultaneously or successively. Additionally, following the publication of some papers discussing databases offered by the National Library of Medicine, such as MeSH or PubMed, the library has used the same channel of information to present data on that subject (Gillikin, 2016; Knecht \& Nelson, 2002). Also using the same communication channel, Elsevier (Meester, Colledge, \& Dyas, 2016) haslikewise confirmed the finding by Franceschini, Maisano, and Mastrogiacomo (2013) of missing links to citations and the finding, recently presented by our research group, of duplicate papers (Valderrama-Zurián, Aguilar-Moya, Melero-Fuentes,\& Aleixandre-Benavent, 2015). It has also been confirmed that such errors will continue to occur because the possiblesolutions would generate a greater number of incorrect citation links among bibliographic records and accidental removal of non-duplicate items than currently exist.

Hence, Scopus and Web of Science Core Collection databases should consider the following amendments:

1-Database indexing visibility: The records should include a visible field indicating the inclusion date in the database and an updated or modified date when required as well as an accompanying description of the modification. The database should also identify possible duplicate records. 
2-Visibility in communication among database and users: Create a formal public forum in which considerations or user questions and answers from the database are registered. Such a forum not only will improve the reliability of the database, but users (namely researchers, research organizations, and research assessment agencies, among others) will also be able to contribute more information to a given subject and read database answers. Following Franceschini, Maisano, and Mastrogiacomo (2016b), it would be useful to learn to what extent error corrections in these databases are initiated by database administrators or are based on the reports of authors or users.Considering these two proposed amendments would improve (i) the rigour and quality of how the named databases treat data and (ii) the reliability of data for providing indicators that are used, among other purposes, to facilitate competitive processes for awarding funding and grants and for selecting researchers and university faculty.

Juan-Carlos Valderrama-Zurián

Dpto. de Historia de la Ciencia y Documentación, Universidad de Valencia, Spain

David Melero-Fuentes

Instituto de Documentación y Tecnologías de la Información, Universidad Católica de Valencia "San Vicente Mártir", Plaza Cisneros 4, Spain

Rafael Aleixandre-Benavent

Ingenio (CSIC-UPV) \& UISYS-Universidad de Valencia, Spain 